\title{
Synchronous mechanical ventilation of the neonate
}

\author{
M SOUTH AND C J MORLEY \\ Department of Paediatrics, University of Cambridge, Addenbrooke's Hospital, Cambridge
}

SUMmARY We describe a simple technique of neonatal ventilation that induces the neonate to breathe in synchrony with the ventilator. This preserves the beneficial effects of spontaneous respiration (unlike therapeutic paralysis) and reduces potentially harmful interactions between baby and ventilator. The technique uses measurements of the neonate's own respiratory timing.

Unless paralysed or very ill, most ventilated neonates continue to breathe, but often out of step with the ventilator. ${ }^{1}$

The neonate's respiratory efforts can make a considerable contribution to ventilation but can also reduce the efficacy of ventilator treatment and increase the risk of pneumothorax. ${ }^{2}$

Spontaneous inspiration or expiration during the 'deflation' phase of a constant flow ventilator should be harmless. It is when spontaneous expiration is active and takes place during the 'inflation' phase of the ventilator that the baby can reduce the tidal volume delivered by the ventilator and when pneumothorax is most likely to occur. ${ }^{2}$ We have used the term 'active' in this paper to represent the pattern described as such in previous work but acknowledge the fact that an active component to expiration in this situation awaits confirmation.

Past attempts to deliver synchronous mechanical ventilation have relied on devices triggered by the patient. ${ }^{3}$ This is technically difficult in the preterm neonate as it relies on detecting small fluctuations in airway pressure generated by the patient. It is difficult to produce a ventilator sensitive enough, or with short enough response times, to produce phase synchrony. ${ }^{4}$ Others have ventilated babies with respiratory rates that crudely approximate to the spontaneous respiratory rates of the baby, but they have not studied the patterns of interaction between baby and ventilator. ${ }^{56}$ We have observed that many babies attempt to maintain their own respiratory pattern when subject to mechanical ventilation. In some babies respiratory reflexes are observed that modify this pattern during certain ventilator breaths. $^{7}$

We see potentially harmful asynchronous respiration occurring in one of two ways. Firstly, the neonate may breathe with a respiratory rate different to the ventilator or with an irregular rhythm.
Secondly, the neonate may breathe at the same rate as the ventilator but with inspiration and expiration out of phase. Both patterns result in spontaneous expiration during the inflation phase of the ventilator.

When the neonate and the mechanical ventilator are 'breathing' at the same rate they are 'rate matched'. When the inflation and deflation phases of the ventilator coincide exactly with those of the baby they have 'phase synchrony'. When the ventilator and baby are breathing exactly in time with each other they are therefore rate matched and in phase synchrony. We have investigated the effects of matching the intrinsic respiratory timing of the baby with the ventilator settings.

\section{Patient and methods}

We describe the technique in a baby of 32 weeks' gestation, weighing $1800 \mathrm{~g}$, ventilated as treatment for respiratory distress syndrome, using the Sechrist Model IV-100B infant ventilator. We have successfully used the technique in babies of various gestational ages, ventilated for respiratory distress syndrome and other conditions, employing the Sechrist, Bourns BP200, and SLE Newborn 250 infant ventilators. The Sechrist ventilator has the advantage that inflation and deflation times can be directly and independently set on the ventilator without reference to calculations involving rate and inspiratory:expiratory ratio. It also maintains constant 'peak', 'end', and 'mean' airway pressures at physiological respiratory rates. The technique could be used, however, with any time cycled ventilator that can deliver high ventilation rates and maintain airway pressures at these rates.

The baby's own respiratory efforts were continuously recorded using a pneumatic capsule device (the MR10 Respiration Monitor, Graseby Dynamics 
Ltd). When taped in the sub-xiphisternal position this provides a signal qualitatively representing the baby's spontaneous respiratory efforts with no interference from ventilator inflations as it differentiates ventilator from spontaneous breaths by the presence of xiphisternal retraction. ${ }^{8}$ A similar signal could be obtained, more invasively and with greater difficulty, from measurement of oesophageal pressure or diaphragmatic electromyogram. This signal was used to measure the baby's own inspiratory and expiratory times and also, by comparing it with the ventilator circuit pressure signal, to show the time relation between spontaneous breaths and ventilator inflations.

A pneumotachograph in series with the endotracheal tube allowed measurement of gas flow in and out of the lungs. The flow signal was electronically intregrated to give tidal volume. This has been used to show the effect of the ventilator and the baby's own respirations on gas flow and tidal volume. Ventilator circuit pressure was measured at the proximal end of the endotracheal tube.

The signals were recorded on a Gould 2600S, rectilinear, six channel paper chart recorder, with a full scale deflection frequency response of $50 \mathrm{~Hz}$, at a paper speed of $25 \mathrm{~mm}$ per second. Pressure transducers and pneumotachograph were produced by Mercury Electronics Ltd and the electronic integrator used to produce tidal volume signals was from the Gould 4600 series.

\section{Results}

A polygraphic record from the ventilated baby at 4 hours of age is shown in Figure 1.
The baby was receiving mechanical ventilation as follows: rate 40 breaths/minute (inflation time 0.48 seconds, deflation time 1.02 seconds). Peak pressure was $32 \mathrm{~cm} \mathrm{H}_{2} \mathrm{O}$ and end pressure $4 \mathrm{~cm} \mathrm{H}_{2} \mathrm{O}$, with inspired oxygen $=80 \%$. Arterial blood gases were within the normal ranges.

It can be seen from the capsule trace that the baby was breathing irregularly and was therefore neither rate matched nor in phase synchrony with ventilator cycles. Flow and tidal volume traces showed that the baby's respiratory efforts were disturbing smooth tidal ventilation. Thus tidal volumes were erratic and bore little relation to ventilator timing. During most ventilator inflation phases, gas flow down the endotracheal tube was stopped before the end of the peak pressure plateau. During the first ventilator inflation shown in this section, gas was seen to flow up the endotracheal tube against the peak inflation pressure. Analysis of the capsule signal showed spontaneous expiration at this point, suggesting active expiration.

Having observed this pattern of asynchronous ventilation, we attempted to induce synchrony. The ventilator was switched from intermittent positive pressure ventilation to provide continuous positive airway pressure, with the baby breathing spontaneously for several seconds while recordings were made. From this a period of regular respiration was chosen and the mean inspired and expired times from five spontaneous breaths were calculated from the pneumatic capsule trace. In the case of this baby the calculated times were inspired time 0.28 seconds and expired time 0.32 seconds (rate $=100$ breaths/ minute).

On returning to intermittent positive pressure ventilation the calculated inspiratory and expiratory

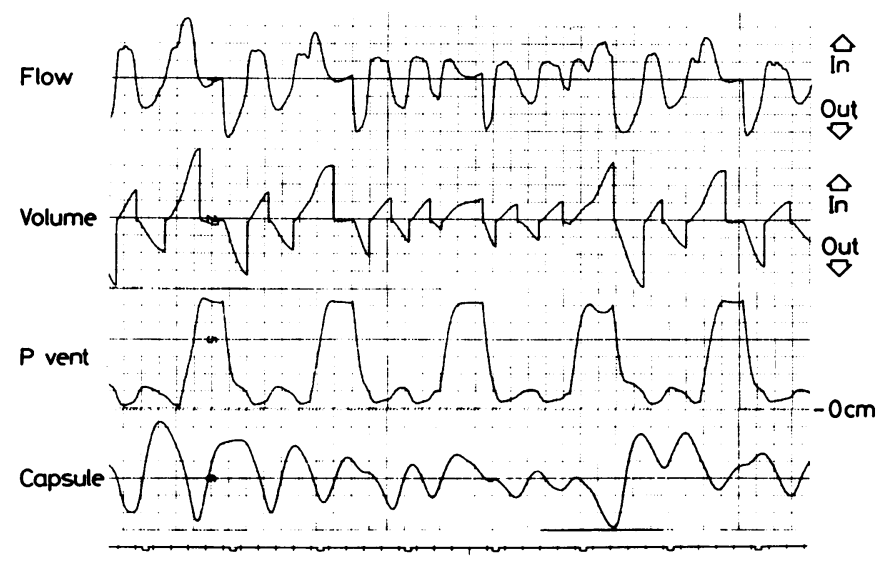

Fig. 1 Asynchronous ventilation at a rate of 40 breaths/min. The top trace shows gas flow and the second trace tidal volume. Deflections above the centre zero line represent flow into the chest and those below the line flow out. The integrator resets to zero at zero flow. The third trace shows the pressure in the ventilator circuit ( $P$ vent $)$ and the fourth trace the signal from the pneumatic capsule, with inspiration represented by a downward deflection on the trace, and expiration by an upward deflection. 
times were used as the ventilator settings. This provided a starting point for synchronisation. This results in rate matching, even if the baby was previously breathing irregularly. It does not always produce perfect phase synchrony, however, as the rate of change in lung volume is increased slightly by the positive inflation pressure. This is shown by analysing the ventilator circuit pressure and capsule traces together and shows whether there is phase synchrony. Small alterations in the ventilator time settings can be made to bring the two into phase. For example, if spontaneous expiration begins before the end of the peak pressure plateau the ventilator inflation time can be reduced until the inspiratory times are equal.

Figure 2 shows phase synchrony achieved in the same baby with inflation and deflation times both 0.3 seconds. There is rate matching and almost perfect phase synchrony; chaotic tidal volumes and episodes of active expiration against ventilator inflation are no longer apparent. Notice how smooth the pneumatic capsule trace has become. It was possible to reduce peak ventilator pressure to $23 \mathrm{~cm}$ $\mathrm{H}_{2} \mathrm{O}$ while keeping arterial blood gases within the normal range.

Figure 3 shows tidal volume and pneumatic capsule signals during two periods each of about half an hour. This shows that the patterns of interaction described are not brief episodes produced by chance but are continuous. Once achieved, synchronous ventilation has persisted for many hours in some babies. In the lower record a period of asynchronous ventilation is seen, at the right hand end of the trace, which followed disturbance for a nursing procedure (rectal thermometer inserted at time indicated by arrow). When left undisturbed the baby is seen to settle back quickly into synchrony.

By 48 hours of age the lung disease had improved and ventilator pressures had been reduced (peak 18 and end $3 \mathrm{~cm} \mathrm{H}_{2} \mathrm{O}$ ), as had inspired oxygen concentration. The baby had remained well synchronised with mechanical inflation and was ready to be weaned from the ventilator. Instead of arbitrary stepwise reductions in ventilator rate, as is often used in rate weaning, the ventilator was set to allow for one complete spontaneous breath and one ventilator synchronised breath, producing $2 \cdot 1$ rate matching between baby and ventilator (Fig. 4). There remained good phase synchrony during the concurrent breaths. This was produced using an inflation time of 0.34 seconds and a deflation time of 1.16 seconds. These figures were obtained from a short period of spontaneous breathing on continuous positive airway pressure, setting the inflation time on the ventilator to that taken spontaneously and the deflation time to that calculated to allow for one full spontaneous respiratory cycle plus expiration from the 'assisted' inflation. As before, it may be necessary to make small alterations in ventilator times to produce exact phase synchrony.

On 36 occasions when babies were breathing? asynchronously with the ventilator, at rates of 75 per

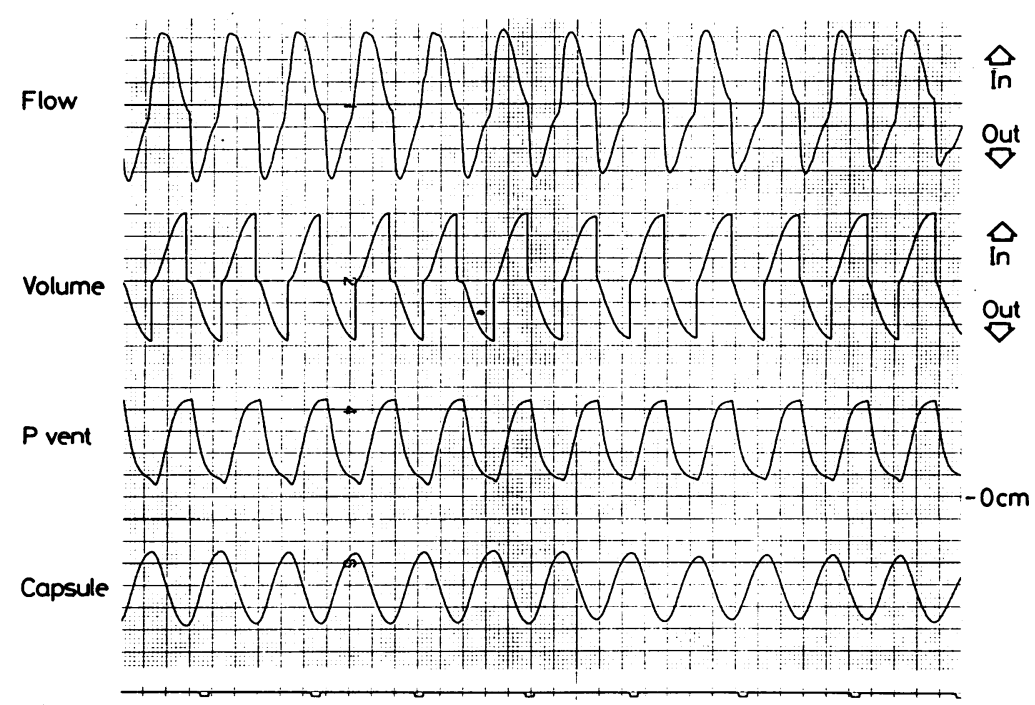

Fig. 2 Established synchronous ventilation at a rate of 100 breaths/ min. See Figure 1 for details of signals. 

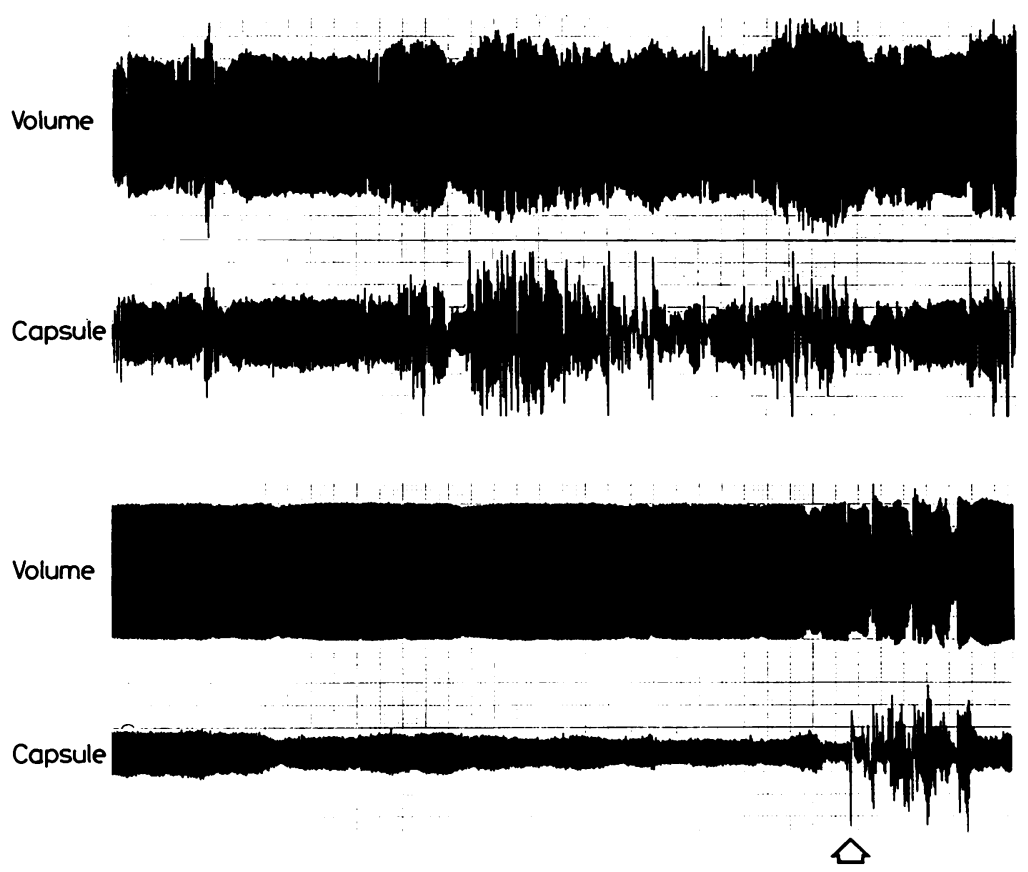

Flow

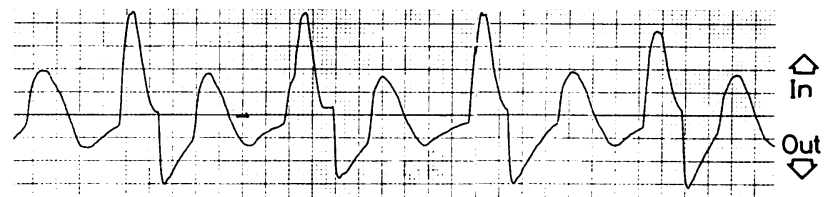

Volume

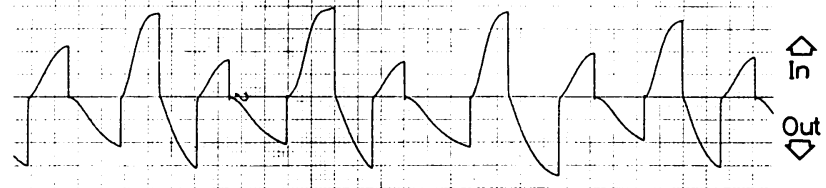

$P$ vent

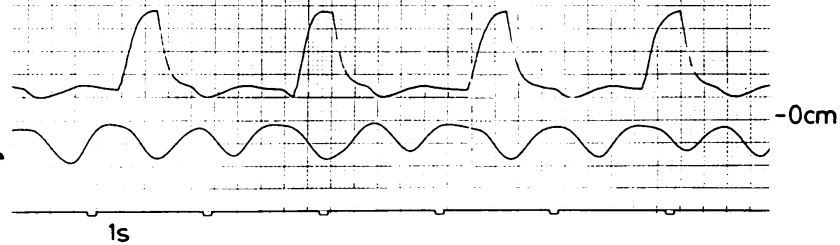

Fig. 3 Tidal volume and pneumatic capsule signals during two half hour periods. The upper pair of traces show the tidal volume and capsule signals during the earlier period of asynchronous ventilation (as in Figure 1) and the lower pair during synchronous ventilation (as in Figure 2). The arrow represents the time of a nursing procedure.
Fig. 4 Rate matching of 2:1 (baby:ventilator) during weaning from ventilator. See Figure 1 for details of signals. minute or less, the degree of synchrony and the amount of expiration during ventilator inflation was improved in all cases by this technique. On most occasions stable synchronous ventilation was established, but in two babies, who were particularly irritable, synchrony was only maintained for short periods of up to 30 minutes. One of these babies was sedated with intravenous diazepam for clinical reasons and was noted thereafter to establish stable prolonged synchrony. 
We have now synchronously ventilated over 20 babies for their entire course on the ventilator as treatment for respiratory distress syndrome. There has been one case of pneumothorax, in a baby ventilated with peak ventilator pressure of $40 \mathrm{~cm}$ $\mathrm{H}_{2} \mathrm{O}$, who had severe 'persistent transitional circulation' in association with respiratory distress syndrome.

\section{Discussion}

We have shown a technique that may be used in babies that breathe asynchronously while receiving mechanical ventilation-that is, babies who are often said to 'fight' the ventilator. In most babies the technique results in smooth synchronous ventilation with few episodes where the baby expires during the inflation phase of the mechanical ventilation.

The method uses a simple idea that takes as a starting point for ventilator settings the spontaneous respiratory timing of the baby and makes small adjustments, if necessary, to result in synchronous delivery of mechanical inflations. It is not surprising that the ventilator timing required to produce synchrony may differ slightly from that found during spontaneous breathing on continuous positive airway pressure as the inspiratory and expiratory time constants and the rate of change in lung volume are likely to be affected by the different tidal volumes and the presence of the inflating pressure.

The technique relies on making measurements of the baby's own respiratory timing. In this paper we have included signals from a pneumotachograph in the endotracheal tube to show the consequences, in terms of tidal volume, of the interaction of the ventilatory efforts of the baby and the ventilator. This should not be necessary in applying the technique clinically as all the information could be obtained from the non-invasively acquired signals of ventilator circuit pressure and the pneumatic capsule.

It would be simpler if a rule of thumb could be devised for ventilator time settings that are likely to produce synchrony as this would dispense with the need for measurements from the baby. Unfortunately, spontaneous respiratory times vary with a number of factors-for example, gestation, ${ }^{9}$ severity of respiratory distress syndrome, age, and size of endotracheal tube. It may be that some of these factors would need to be considered in predicting the best times to use. Our preliminary evidence suggests that for preterm neonates with respiratory distress syndrome in the first 24-48 hours of age, inflation times in the range $0 \cdot 28-0.35$ and expired times in the range $0.35-0.45$ are most likely to be suitable. The times required will probably vary in any given baby as the respiratory time constants follow changes in respiratory compliance and the course of the respiratory distress syndrome.

Recent work failed to show that the arbitrarily chosen rates of 60 or 120 breaths per minute would consistently produce synchrony in those babies previously found to be actively expiring against the ventilator at rates of 30 to 40 . At the higher rates, however, the amount of active expiration was reduced by $88 \%$. (Greenough A, Morley CJ, Pool J. Are fast rates and alternative to paralysis. Presented at the European Society for Pediatric Research, 1985:abstract 145.)

We have not studied the influence of such factors as blood gases, ventilator pressures, or neurological state on the development of synchronous ventilation, but these are likely to be important.

Does synchronous ventilation offer any advantages? The fact that it prevents expiration against ventilator inflation, which has been shown to be a factor in the production of pneumothoraces, ${ }^{2}$ should be of importance. It may reduce the need for therapeutic paralysis. Heicher $e t$ al and Pohlandt $e t$ $a l$ have shown a reduced incidence of pneumothorax in babies ventilated at fast rates with short inflation times. ${ }^{10}$ (Pohlandt F, Bernsau U, Feilen KD, et al. Reduction of barotrauma in ventilated neonates byc increase in ventilation frequency. Presented at the European Society for Pediatric Research, 1985:abstract 144.) This would seem to be at variance with theories suggesting that high rates produce 'inadvertent positive end expiratory pressure' at distal airway level. ${ }^{11}{ }^{12}$ These theories, based on respiratory time constant analysis, only consider the static lung and ignore the effects of spontaneous respiratory effort on respiratory time constants and rate of change in lung volume. It may be that in the studies of Heicher $e t$ al and Pohlandt $e$ t al there were fewer pneumothoraces in the fast rate groups because more babies were breathing in synchrony with their ventilators, as the fast rate groups received ventilator timing similar to that we use to produce synchrony. Unfortunately, these factors were not studied. In our babies the incidence of pneumothorax has been low so far (one out of over 20 babies), but this observation needs further investigation by means of a prospective, randomised, controlled trial.

There are theoretical advantages from synchronous mechanical ventilation in terms of cardiac output. Mean intrathoracic pressure is higher during asynchronous controlled ventilation than during synchronous assisted ventilation, ${ }^{13}$ and this may reduce cardiac venous filling. Asynchronous controlled ventilation results in random swings in intra-atrial pressure, whereas synchronous ventila- 
tion results in a more physiological haemodynamic situation. ${ }^{14}$ This may also have implications for the babies at risk from intracranial haemorrhage. ${ }^{15}$

Preliminary evidence we have from the babies we have studied shows an upward trend in minute ventilation with a consequent improvement in blood gases when the baby is breathing in synchrony with its ventilator but not when he is breathing in asynchrony. There may also be advantageous consequences of synchronous ventilation in terms of energy expenditure. Anecdotally, our nursing staff report that the baby who is breathing asynchronously is usually fractious and often struggles a lot, while the baby who is synchronously ventilated is often quieter and 'easier to nurse'.

We have noticed several other phenomena during synchronous ventilation. The baby who was breathing irregularly, both during asynchronous ventilation and during short periods of spontaneous breathing on continuous positive airway pressure, often breathed regularly and continuously once established on synchronous ventilation. The baby who was well synchronised on mechanical ventilation rapidly reestablished phase synchrony after a period with intermittent positive pressure ventilation switched off, no matter at what phase of the spontaneous respiratory cycle ventilation was restarted. These phenomena of rate and phase entrainment have been observed in anaesthetised cats subject to mechanical ventilation ${ }^{16}$ and are probably vagally mediated.

The process of rate weaning from the ventilator can be modified to produce synchronous ventilation as described. It is possible to produce various patterns of synchrony-for example, 2:1 and 3:1 rate matching between baby and ventilator. This may well provide for a more logical method of weaning, avoiding episodes of active expiration against ventilator inflation, than the arbitrary stepwise reductions in rate that are often made.

Studies of ventilator regimens that ignore the effects of spontaneous respiration may fail to recognise important factors. Ventilated babies, even those with the same disease, do not form a single homogenous group as they may be paralysed, apnoeic, or breathing synchronously with the ventilator.

We have shown that it is possible to induce synchronous ventilation by this technique. Whether it does offer the potential advantages outlined awaits further investigation.
Gandy for their encouragement and advice and the nursing staff of the neonatal unit at the Rosie Maternity Hospital.

This work was funded by the University of Cambridge Baby Research Fund.

\section{References}

1 Greenough A, Morley CJ, Davis JA. Interaction of spontaneous respiration with artificial ventilation in preterm babies. Pediatrics 1983;103:769-73.

2 Greenough A, Morley CJ, Wood S, Davis JA. Pancuronium prevents pneumothorax in ventilated premature babies who actively expire against positive pressure inflation. Lancet 1984;i:1-3.

${ }^{3}$ Kirby RR. Design of mechanical ventilators: assisted and controlled ventilation. In: Thibeault DW, Gregory GA, eds. Neonatal pulmonary care. Addison Wesley Publishing, 1979:157-9.

4 Epstein RA. The sensitivities and response time of ventilatory assistors. Anesthesiology 1971;34:321-6.

5 Adamson TM, Collins LM, Hawker JM, Reynolds EOR, Strang LB. Mechanical ventilation in newborn infants with respiratory failure. Lancet 1968;ii:227-31.

${ }^{6}$ Bland RD, Kim MH, Light MJ, Woodson JL. High frequency mechanical ventilation of low-birthweight infants with respiratory failure from hyaline membrane disease: $92 \%$ survival. Pediatr Res 1977;11:531.

${ }^{7}$ Greenough A, Morley CJ, Davis JA. Respiratory reflexes in ventilated premature babies. Early Hum Dev 1983;8:65-75.

${ }^{8}$ South M, Morley CJ. A technique for monitoring spontancous respiration in the ventilated neonate. Arch Dis Child 1986;61:291-4.

9 Curzi-Dascalova L, Lebrun F, Korn G. Respiratory frequency according to sleep states and age in normal premature infants: a comparison with full term infants. Pediatr Res 1983:17:152-6.

${ }^{10}$ Heicher DA, Kasting DS, Harrod J. Prospective clinical comparison of two methods for mechanical ventilation of neonates: rapid rate and short inspiratory time versus slow rate and long inspiratory time. J Pediatr 1981;98:957-61.

"Donahue LA, Thibeault DW. Alveolar gas trapping and ventilator therapy in infants. Respiratory Therapy 1979;3:47-51.

12 Weigl J. The infant lung: a case against high respiratory rates in controlled neonatal ventilation. Respiratory Therapy 1973:3: 57-71.

13 Owen-Thomas JB, Ulan OA, Swyer PR. The effect of varying inspiratory gas flow rate on arterial oxygenation during intermittent positive pressure ventilation in respiratory distress syndrome. Br J Anaesth 1968;40:493-501.

${ }^{14}$ Llewellyn MA. Aspects of the pathophysiology of artificial mechanical ventilation in the newborn infant. London: University of London, 1971. (MD Thesis.)

15 Perlman JM, McMenamin JB, Volpe JJ. Fluctuating cerebral blood-flow velocity in respiratory distress syndrome-relationship to the development of intraventricular hemorrhage. $N$ Eng J Med 1983;309:204-9.

16 Petrillo GA, Glass L, Trippenbach T. Phase locking of the respiratory rhythm in cats to a mechanical ventilator. Can $J$ Physiol Pharmacol 1983;61:599-607.

Correspondence to Dr M South, Department of Paediatrics, University of Cambridge, Level E8, Addenbrooke's Hospital, Cambridge, England.

Received 26 June 1986 